\title{
New Meir-Keeler Type Tripled Fixed-Point Theorems on Ordered Partial Metric Spaces
}

\author{
Hassen Aydi $^{1}$ and Erdal Karapinar ${ }^{2}$ \\ ${ }^{1}$ Institut Supérieur d'Informatique et des Technologies de Communication de Hammam Sousse, \\ Université de Sousse, Route GP1 4011, Tunisia \\ ${ }^{2}$ Department of Mathematics, Atilim University, Incek, 06836 Ankara, Turkey
}

Correspondence should be addressed to Erdal Karapınar, erdalkarapinar@yahoo.com

Received 3 February 2012; Accepted 27 March 2012

Academic Editor: Zheng-Guang Wu

Copyright (C) 2012 H. Aydi and E. Karapınar. This is an open access article distributed under the Creative Commons Attribution License, which permits unrestricted use, distribution, and reproduction in any medium, provided the original work is properly cited.

In this paper, we prove some new Meir-Keeler type tripled fixed-point theorems on a partially ordered complete partial metric space. Also, as application, some results of integral type are given.

\section{Introduction and Preliminaries}

In the last century, the theory of fixed points has appeared as a crucial technique in the study of nonlinear phenomena. Particularly, the tools in fixed-point theory have an application in such diverse fields as biology, chemistry, physics, economics, computer sciences, and engineering.

Recently, fixed-point theorems are considered on partial metric spaces on which selfdistance of some points may not be zero. This phenomenon was discovered by Matthews [1] when he considered the tools of metric spaces in the field of semantics and domain theory in computer science (see, e.g., $[2,3]$ ). After the initial results of Mathews, other papers have been released on partial metric spaces (see e.g., [4-20]).

Another important development is reported in fixed-point theory via ordered metric spaces. Fixed-point theorems in ordered sets were discussed by Ran and Reurings [21]. Subsequently, many results in this direction were given (see, e.g., [22-31]).

In this paper, we combine two recent trends, partial metric spaces and ordered sets, and discuss the existence and uniqueness of some new Meir-Keeler type tripled fixed-point theorems in the context of partially ordered partial metric spaces.

Let $X$ be a nonempty set. A partial metric is a function $p: X \times X \rightarrow[0, \infty)$ satisfying the following conditions: 
$(P 1)$ if $p(x, x)=p(x, y)=p(y, y)$, then $x=y$,

(P2) $p(x, y)=p(y, x)$,

(P3) $p(x, x) \leq p(x, y)$,

(P4) $p(x, z)+p(y, y) \leq p(x, y)+p(y, z)$,

for all $x, y, z \in X$. Then, $(X, p)$ is called a partial metric space.

If $p$ is a partial metric $p$ on $X$, then the function $d_{p}: X \times X \rightarrow[0, \infty)$ given by

$$
d_{p}(x, y)=2 p(x, y)-p(x, x)-p(y, y)
$$

is a metric on $X$. Each partial metric $p$ on $X$ generates a $T_{0}$ topology $\tau_{p}$ on $X$ with a base of the family of open $p$-balls $\left\{B_{p}(x, \varepsilon): x \in X, \varepsilon>0\right\}$, where $B_{p}(x, \varepsilon)=\{y \in X: p(x, y)<p(x, x)+\varepsilon\}$ for all $x \in X$ and $\varepsilon>0$. Similarly, closed $p$-ball is defined as $B_{p}[x, \varepsilon]=\{y \in X: p(x, y) \leq$ $p(x, x)+\varepsilon\}$. For more details, see $[1,5]$.

Definition 1.1 (see, e.g., $[1,5,15])$. Let $(X, p)$ be a partial metric space.

(i) A sequence $\left\{x_{n}\right\}$ in $X$ converges to $x \in X$ whenever $\lim _{n \rightarrow \infty} p\left(x, x_{n}\right)=p(x, x)$.

(ii) A sequence $\left\{x_{n}\right\}$ in $X$ is called Cauchy whenever $\lim _{n, m \rightarrow \infty} p\left(x_{n}, x_{m}\right)$ exists (and finite).

(iii) $(X, p)$ is said to be complete if every Cauchy sequence $\left\{x_{n}\right\}$ in $X$ converges, with respect to $\tau_{p}$, to a point $x \in X$, that is, $\lim _{n, m_{\rightarrow} \infty} p\left(x_{n}, x_{m}\right)=p(x, x)$.

Lemma 1.2 (see, e.g., $[1,5,15])$. Let $(X, p)$ be a partial metric space.

(a) A sequence $\left\{x_{n}\right\}$ is Cauchy if and only if $\left\{x_{n}\right\}$ is a Cauchy sequence in the metric space $\left(X, d_{p}\right)$.

(b) $(X, p)$ is complete if and only if the metric space $\left(X, d_{p}\right)$ is complete. Moreover,

$$
\lim _{n \rightarrow \infty} d_{p}\left(x, x_{n}\right)=0 \Longleftrightarrow \lim _{n \rightarrow \infty} p\left(x, x_{n}\right)=\lim _{n, m \rightarrow \infty} p\left(x_{n}, x_{m}\right)=p(x, x) .
$$

Lemma 1.3 (see, e.g., $[4,15,16])$. Let $(X, p)$ be a partial metric space. Then,

(A) if $p(x, y)=0$, then $x=y$,

(B) if $x \neq y$, then $p(x, y)>0$.

Remark 1.4. If $x=y, p(x, y)$ may not be 0 .

Lemma 1.5 (see, e.g., $[4,15,16])$. Let $x_{n} \rightarrow z$ as $n \rightarrow \infty$ in a partial metric space $(X, p)$ where $p(z, z)=0$. Then, $\lim _{n \rightarrow \infty} p\left(x_{n}, y\right)=p(z, y)$ for every $y \in X$.

$(X, p, \leq)$ is called a partially ordered partial metric space if $(X, \leq)$ is a partially ordered set and $(X, p)$ is a partial metric space. Further, if $(X, p)$ is a complete partial metric space, then $(X, p, \leq)$ is called a partially ordered complete partial metric space. Hereafter, we assume that $X \neq \emptyset$ and we use the notation

$$
X^{k}=\underbrace{X \times X \times \cdots \times X}_{k \text {-many }} .
$$


Also, take the mapping $P: X^{3} \times X^{3} \rightarrow[0,+\infty)$ such that

$$
P(\mathbf{x}, \mathbf{y}):=\max \left\{p\left(x_{1}, y_{1}\right), p\left(x_{2}, y_{2}\right), p\left(x_{3}, y_{3}\right)\right\},
$$

where $\mathbf{x}=\left(x_{1}, x_{2}, x_{3}\right)$ and $\mathbf{y}=\left(y_{1}, y_{2}, y_{3}\right) \in X^{3}$.

Let $(X, \leq)$ be a partially ordered set. We consider the following partial order (also denoted by $\leq$ ) on the product space $X^{3}$ :

$$
(u, v, w) \leq(x, y, z) \quad \text { iff } x \geq u, y \leq v, z \geq w,
$$

where $(u, v, w),(x, y, z) \in X^{3}$. Moreover, we say that $(x, y, z)$ is equal to $(u, v, r)$ if and only if $x=u,=v$, and $z=r$. In the sequel, we need the following definitions.

Definition 1.6 (see [32]). Let $(X, \leq)$ be a partially ordered set and $F: X^{3} \rightarrow X$ a given mapping. We say that $F$ has the mixed monotone property if $F(x, y, z)$ is monotone nondecreasing in $x$ and $z$, and it is monotone nonincreasing in $y$, that is, for any $x, y, z \in X$,

$$
\begin{aligned}
& x_{1}, x_{2} \in X, x_{1} \leq x_{2} \Longrightarrow F\left(x_{1}, y, z\right) \leq F\left(x_{2}, y, z\right) \\
& y_{1}, y_{2} \in X, y_{1} \leq y_{2} \Longrightarrow F\left(x, y_{1}, z\right) \geq F\left(x, y_{2}, z\right) \\
& z_{1}, z_{2} \in X, z_{1} \leq z_{2} \Longrightarrow F\left(x, y, z_{1}\right) \leq F\left(x, y, z_{2}\right)
\end{aligned}
$$

Definition 1.7 (see [32]). An element $(x, y, z) \in X^{3}$ is called a tripled fixed point of $F: X^{3} \rightarrow X$ if

$$
F(x, y, z)=x, \quad F(y, x, y)=y, \quad F(z, y, x)=z \text {. }
$$

Berinde and Borcut [32] proved the following theorem.

Theorem 1.8. Let $(X, \leq)$ be a partially ordered set and $(X, d)$ a complete metric space. Let $F: X^{3} \rightarrow$ $X$ be a mapping having the mixed monotone property on $X$. Assume that there exist constants $a, b, c \in$ $[0,1)$ such that $a+b+c<1$ for which

$$
d(F(x, y, z), F(u, v, w)) \leq a d(x, u)+b d(y, v)+c d(z, w)
$$

for all $x \geq u, y \leq v$ and $z \geq w$. Assume that $X$ has the following properties:

(i) if a nondecreasing sequence $x_{n} \rightarrow x$, then $x_{n} \leq x$ for all $n$,

(ii) if a nonincreasing sequence $y_{n} \rightarrow y$, then $y_{n} \geq y$ for all $n$.

If there exist $x_{0}, y_{0}, z_{0} \in X$ such that

$$
x_{0} \leq F\left(x_{0}, y_{0}, z_{0}\right), \quad y_{0} \geq F\left(y_{0}, x_{0}, y_{0}\right), \quad z_{0} \leq F\left(z_{0}, y_{0}, x_{0}\right),
$$

then there exist $x, y, z \in X$ such that

$$
F(x, y, z)=x, \quad F(y, x, y)=y, \quad F(z, y, x)=z .
$$


Recently, Theorem 1.8 is extended to cone metric spaces by Rao and Kishore [33]. On the other hand, very recently, Aydi et al. [34] introduced the following concepts.

Definition 1.9 (see [34]). Let $(X, \leq)$ be a partially ordered set and $F: X^{3} \rightarrow X$. We say that $F$ has the mixed strict monotone property if, for any $x, y, z \in X$,

$$
\begin{aligned}
& x_{1}, x_{2} \in X, x_{1}<x_{2} \Longrightarrow F\left(x_{1}, y, z\right)<F\left(x_{2}, y, z\right) \\
& y_{1}, y_{2} \in X, y_{1}<y_{2} \Longrightarrow F\left(x, y_{1}, z\right)>F\left(x, y_{2}, z\right) \\
& z_{1}, z_{2} \in X, z_{1}<z_{2} \Longrightarrow F\left(x, y, z_{1}\right)<F\left(x, y, z_{2}\right)
\end{aligned}
$$

Definition 1.10 (see [34]). Let $(X, d, \leq)$ be a partially ordered metric space. A mapping $F$ : $X^{3} \rightarrow X$ is said to be a generalized Meir-Keeler type contraction if, for any $\varepsilon>0$, there exists a $\delta(\varepsilon)>0$ such that

$$
\varepsilon \leq \max \{d(x, u), d(y, v), d(z, r)\}<\varepsilon+\delta(\varepsilon) \Longrightarrow d(F(x, y, z), F(u, v, r))<\varepsilon,
$$

for all $x, y, z, u, v, r \in X$ with $x \leq u, y \geq v$ and $z \leq r$.

In the following, we consider the partial case of Definition 1.10 and we introduce the following.

Definition 1.11. Let $(X, p, \leq)$ be a partially ordered partial metric space. A mapping $F: X^{3} \rightarrow$ $X$ is said to be a generalized $p$-Meir-Keeler type contraction if, for any $\varepsilon>0$, there exists a $\delta(\varepsilon)>0$ such that

$$
\varepsilon \leq \max \{p(x, u), p(y, v), p(z, r)\}<\varepsilon+\delta(\varepsilon) \Longrightarrow p(F(x, y, z), F(u, v, r))<\varepsilon,
$$

for all $x, y, z, u, v, r \in X$ with $x \leq u, y \geq v$ and $z \leq r$.

Remark 1.12. It is immediate to show that if $F: X^{3} \rightarrow X$ is a generalized $p$-Meir-Keeler type contraction, then

$$
p(F(x, y, z), F(u, v, r))<\max \{p(x, u), p(y, v), p(z, r)\}
$$

for all $x, u, y, v, z, r, \in X$ with $x<u, y \geq v, z<r$ or $x \leq u, y>v, z \leq r$.

Proposition 1.13. Let $(X, p, \leq)$ be a partially ordered partial metric space and $F: X^{3} \rightarrow X$ a given mapping. If (1.8) is satisfied, then $F$ is a generalized $p$-Meir-Keeler type function.

Proof. Assume that (1.8) is satisfied. For all $\varepsilon>0$, one can check that (1.13) is satisfied with $\delta(\varepsilon)=(1 /(a+b+c)-1) \varepsilon$.

In the sequel, we use the following notations given in [34]. Let $\widetilde{F}: X^{3} \rightarrow X^{3}$ be such that, for $a, b, c \in X$,

$$
\widetilde{F}(a, b, c)=(F(a, b, c), F(b, a, b), F(c, b, a))
$$


Let $x_{0}, y_{0}, z_{0} \in X$ be such that

$$
x_{0}<F\left(x_{0}, y_{0}, z_{0}\right), \quad y_{0} \geq F\left(y_{0}, x_{0}, y_{0}\right), \quad z_{0}<F\left(z_{0}, y_{0}, x_{0}\right) .
$$

We consider sequences $\left\{x_{n}\right\},\left\{y_{n}\right\}$, and $\left\{z_{n}\right\}$ such that

$$
\underbrace{\left[\begin{array}{l}
x_{n} \\
y_{n} \\
z_{n}
\end{array}\right]}_{A_{n}}=\underbrace{\left[\begin{array}{l}
F\left(x_{n-1}, y_{n-1}, z_{n-1}\right) \\
F\left(y_{n-1}, x_{n-1}, y_{n-1}\right) \\
F\left(z_{n-1}, y_{n-1}, x_{n-1}\right)
\end{array}\right]}_{\tilde{F}\left(A_{n-1}\right)}=\underbrace{\left[\begin{array}{l}
F^{n}\left(x_{0}, y_{0}, z_{0}\right) \\
F^{n}\left(y_{0}, x_{0}, y_{0}\right) \\
F^{n}\left(z_{0}, y_{0}, x_{0}\right)
\end{array}\right]}_{\widetilde{F^{n}}\left(A_{0}\right)},
$$

for $n=1,2,3, \ldots$

Our first auxiliary result is as follows.

Proposition 1.14. Let $(X, p, \leq)$ be a partially ordered partial metric space, and let $F: X^{3} \rightarrow$ Xbe a given mapping such that the following hypotheses hold:

(i) F has the mixed strict monotone property,

(ii) $F$ is a generalized $p$-Meir-Keeler type function,

(iii) $\exists(x, y, z),(u, v, r) \in X^{3}$ such that $x<u, y \geq v$ and $z<r$.

Then,

$$
P\left(\widetilde{F^{n}}(x, y, z), \widetilde{F^{n}}(u, v, r)\right) \longrightarrow 0, \quad \text { as } n \longrightarrow+\infty
$$

Proof. Let $(x, y, z)=\left(x_{0}, y_{0}, z_{0}\right)$ and $(u, v, r)=\left(u_{0}, v_{0}, r_{0}\right)$. We show that

$$
\begin{aligned}
& x_{n}=F^{n}\left(x_{0}, y_{0}, z_{0},\right)<F^{n}\left(u_{0}, v_{0}, r_{0}\right)=u_{n}, \\
& y_{n}=F^{n}\left(y_{0}, x_{0}, y_{0}\right)>F^{n}\left(v_{0}, u_{0}, v_{0}\right)=v_{n}, \quad \forall n=1,2, \ldots, \\
& z_{n}=F^{n}\left(z_{0}, y_{0}, x_{0}\right)<F^{n}\left(r_{0}, v_{0}, u_{0}\right)=r_{n},
\end{aligned}
$$

with $F=F^{1}$.

Due to the fact that $F$ has the mixed strict monotone property, together with the assumption that $x<u, y \geq v$ and $z<r$, we obtain

$$
\begin{aligned}
x_{1} & =F(x, y, z)=F\left(x_{0}, y_{0}, z_{0}\right)<F\left(u_{0}, y_{0}, z_{0}\right) \\
& \Longrightarrow F\left(x_{0}, y_{0}, z_{0}\right)<F\left(u_{0}, v_{0}, z_{0}\right) \\
& \Longrightarrow F\left(x_{0}, y_{0}, z_{0}\right)<F\left(u_{0}, v_{0}, r_{0}\right)=u_{1} .
\end{aligned}
$$

Analogously, we have

$$
y_{1}=F\left(y_{0}, x_{0}, y_{0}\right)>F\left(v_{0}, u_{0}, v_{0}\right)=v_{1}, \quad z_{1}=F\left(z_{0}, y_{0}, x_{0}\right)<F\left(r_{0}, v_{0}, u_{0}\right)=r_{1}
$$


Thus, (1.19) holds for $n=1$. By using the same arguments, we show that (1.19) holds also for $n=2$. In fact,

$$
\begin{aligned}
x_{2} & =F^{2}\left(x_{0}, y_{0}, z_{0}\right)=F\left(x_{1}, y_{1}, z_{1}\right) \\
& =F\left(F\left(x_{0}, y_{0}, z_{0}\right), F\left(y_{0}, x_{0}, y_{0}\right), F\left(z_{0}, y_{0}, x_{0}\right)\right) \\
& <F\left(F\left(u_{0}, v_{0}, r_{0}\right), F\left(y_{0}, x_{0}, y_{0}\right), F\left(z_{0}, y_{0}, x_{0}\right)\right) \\
& <F\left(F\left(u_{0}, v_{0}, r_{0}\right), F\left(v_{0}, u_{0}, v_{0}\right), F\left(z_{0}, y_{0}, x_{0}\right)\right) \\
& <F\left(F\left(u_{0}, v_{0}, r_{0}\right), F\left(v_{0}, u_{0}, v_{0}\right), F\left(r_{0}, v_{0}, u_{0}\right)\right) \\
& =F^{2}\left(u_{0}, v_{0}, r_{0}\right)=F\left(u_{1}, v_{1}, r_{1}\right)=u_{2} .
\end{aligned}
$$

Similarly, we find

$$
y_{2}=F^{2}\left(y_{0}, x_{0}, y_{0}\right) \geq F^{2}\left(v_{0}, u_{0}, v_{0}\right)=v_{2}, \quad z_{2}=F^{2}\left(z_{0}, y_{0}, x_{0}\right)<F^{2}\left(r_{0}, v_{0}, u_{0}\right)=r_{2}
$$

Inductively, we get that (1.19) holds.

By Remark 1.12, together with (1.19), we have

$$
\begin{aligned}
p\left(x_{n+2}, u_{n+2}\right) & =p\left(F^{n+2}\left(x_{0}, y_{0}, z_{0}\right), F^{n+2}\left(u_{0}, v_{0}, r_{0}\right)\right) \\
& =p\left(F\left(x_{n+1}, y_{n+1}, z_{n+1}\right), F\left(u_{n+1}, v_{n+1}, r_{n+1}\right)\right) \\
& <\max \left\{p\left(x_{n+1}, u_{n+1}\right), p\left(y_{n+1}, v_{n+1}\right), p\left(z_{n+1}, r_{n+1}\right)\right\}, \\
p\left(z_{n+2}, r_{n+2}\right) & =p\left(F^{n+2}\left(z_{0}, y_{0}, x_{0}\right), F^{n+2}\left(r_{0}, v_{0}, u_{0}\right)\right) \\
& =p\left(F\left(z_{n+1}, y_{n+1}, x_{n+1}\right), F\left(r_{n+1}, v_{n+1}, u_{n+1}\right)\right) \\
& <\max \left\{p\left(z_{n+1}, r_{n+1}\right), p\left(y_{n+1}, v_{n+1}\right), p\left(x_{n+1}, u_{n+1}\right)\right\} \\
p\left(y_{n+2}, v_{n+2}\right) & =p\left(F^{n+2}\left(y_{0}, x_{0}, y_{0}\right), F^{n+2}\left(v_{0}, u_{0}, v_{0}\right)\right) \\
& =p\left(F\left(y_{n+1}, x_{n+1}, y_{n+1}\right), F\left(v_{n+1}, u_{n+1}, v_{n+1}\right)\right) \\
& <\max \left\{p\left(y_{n+1}, v_{n+1}\right), p\left(x_{n+1}, u_{n+1}\right), p\left(y_{n+1}, v_{n+1}\right)\right\} \\
& \leq \max \left\{p\left(z_{n+1}, r_{n+1}\right), p\left(y_{n+1}, v_{n+1}\right), p\left(x_{n+1}, u_{n+1}\right)\right\} .
\end{aligned}
$$

Let $\Delta_{n+1}:=\max \left\{p\left(x_{n+1}, u_{n+1}\right), p\left(y_{n+1}, v_{n+1}\right), p\left(z_{n+1}, r_{n+1}\right)\right\}$. Combining (1.24)-(1.26), we get

$$
\Delta_{n+2}<\Delta_{n+1}, \quad \forall n=1,2 \ldots
$$

If we denote $B_{n}=\left(u_{n}, v_{n}, r_{n}\right)$, then, by definition of the partial metric $P$ and (1.27), we have

$$
P\left(A_{n+2}, B_{n+2}\right)<P\left(A_{n+1}, B_{n+1}\right) .
$$


Consequently, the sequence $\left\{t_{n}\right\}=\left\{P\left(A_{n}, B_{n}\right)\right\}$ is decreasing. Hence, $\left\{t_{n}\right\}$ converges, say to $\varepsilon \geq 0$. Clearly, if $\varepsilon=0$, we have finished. Suppose, on the contrary, $\varepsilon>0$. Thus, there exists $k \in\{1,2, \ldots\}$ such that

$$
\varepsilon \leq t_{n}=P\left(A_{n}, B_{n}\right)<\varepsilon+\delta(\varepsilon) \quad \text { for any } n \geq k
$$

In particular, for $n=k$, we have

$$
\varepsilon \leq t_{k}=P\left(A_{k}, B_{k}\right)<\varepsilon+\delta(\varepsilon)
$$

that is equal to

$$
\varepsilon \leq \Delta_{k}<\varepsilon+\delta(\varepsilon)
$$

It follows from (1.19) and the hypothesis (ii) that

$$
p\left(F\left(x_{k}, y_{k}, z_{k}\right), F\left(u_{k}, v_{k}, r_{k}\right)\right)<\varepsilon
$$

which is equivalent to

$$
p\left(x_{k+1}, u_{k+1}\right)<\varepsilon
$$

Moreover, we have

$$
p\left(y_{k+1}, v_{k+1}\right)<\varepsilon, \quad p\left(z_{k+1}, r_{k+1}\right)<\varepsilon
$$

Combining (1.33) and (1.34), we have

$$
\Delta_{k+1}<\varepsilon
$$

Thus, $t_{k+1}=P\left(A_{k+1}, B_{k+1}\right)<\varepsilon$ which is a contradiction with respect to (1.29), and so $\varepsilon=0$. We conclude that

$$
P\left(A_{n}, B_{n}\right)=P\left(\widetilde{F^{n}}(x, y, z), \widetilde{F^{n}}(u, v, r)\right) \longrightarrow 0, \quad \text { as } n \longrightarrow+\infty
$$

Remark 1.15. The previous proposition remains true if, in (iii), we change the assumption

$$
\exists(x, y, z),(u, v, r) \in X^{3} \quad \text { such that } x<u, y \geq v, z<r
$$

with the following

$$
\exists(x, y, z),(u, v, r) \in X^{3} \quad \text { such that } x \leq u, y>v, z \leq r .
$$




\section{Existence of Tripled Fixed Point}

The following theorem is our first main result.

Theorem 2.1. Let $(X, p, \leq)$ be a partially ordered complete partial metric space. Suppose that $X$ has the following properties:

(a) if $\left\{x_{n}\right\}$ is a sequence such that $x_{n+1}>x_{n}$ for each $n=1,2, \ldots$ and $x_{n} \rightarrow x$, then $x_{n}<x$ for each $n=1,2, \ldots$,

(b) if $\left\{y_{n}\right\}$ is a sequence such that $y_{n+1}<y_{n}$ for each $n=1,2, \ldots$ and $y_{n} \rightarrow y$, then $y_{n}>y$ for each $n=1,2, \ldots$.

Assume that $F: X^{3} \rightarrow X$ satisfies the following hypotheses:

(i) F has the mixed strict monotone property,

(ii) $F$ is a generalized $p$-Meir-Keeler type function,

(iii) there exist $x_{0}, y_{0}, z_{0} \in X$ such that

$$
x_{0}<F\left(x_{0}, y_{0}, z_{0}\right), \quad y_{0} \geq F\left(y_{0}, x_{0}, y_{0}\right), \quad z_{0}<F\left(z_{0}, y_{0}, x_{0},\right) .
$$

Then, $F$ has a tripled fixed point, that is, there exist $x, y, z \in X$ such that

$$
F(x, y, z)=x, \quad F(y, x, y)=y, \quad F(z, y, x)=z \text {. }
$$

Also, $p(x, x)=p(y, y)=p(z, z)=0$.

Proof. Let $x_{0}, y_{0}, z_{0} \in X$ be as in (iii). We construct sequences $\left\{x_{n}\right\},\left\{y_{n}\right\}$, and $\left\{z_{n}\right\}$ according to (1.17).

We claim that, for all $n \geq 2$, we have

$$
\begin{aligned}
& \cdots>x_{n}>x_{n-1}>\cdots>x_{1}>x_{0}, \\
& \cdots<y_{n}<y_{n-1}<\cdots<y_{1} \leq y_{0}, \\
& \cdots>z_{n}>z_{n-1}>\cdots>z_{1}>z_{0} .
\end{aligned}
$$

Indeed, we will use a mathematical induction to prove (2.3). Clearly, we have

$$
x_{0}<F\left(x_{0}, y_{0}, z_{0}\right)=x_{1}, \quad y_{0} \geq F\left(y_{0}, x_{0}, y_{0}\right)=y_{1}, \quad z_{0}<F\left(z_{0}, y_{0}, x_{0}\right)=z_{1} \text {. }
$$

Suppose now that the inequalities in (2.3) hold for some $n \geq 2$. By the mixed strict monotone property of $F$, together with (1.17), we have

$$
\begin{aligned}
& x_{n}=F\left(x_{n-1}, y_{n-1}, z_{n-1}\right)<F\left(x_{n}, y_{n}, z_{n}\right)=x_{n+1}, \\
& y_{n}=F\left(y_{n-1}, x_{n-1}, y_{n-1}\right)>F\left(y_{n}, x_{n}, y_{n}\right)=y_{n+1}, \\
& z_{n}=F\left(z_{n-1}, y_{n-1}, x_{n-1}\right)<F\left(z_{n}, y_{n}, x_{n}\right)=z_{n+1} .
\end{aligned}
$$

Thus, (2.3) holds for all $n \geq 2$. 
Mathematical Problems in Engineering

Putting $(x, y, z)=A_{0}$ and $(u, v, r)=A_{1}$ and by Proposition 1.14, we get

$$
P\left(\widetilde{F^{n}}\left(A_{0}\right), \widetilde{F^{n}}\left(A_{1}\right)\right) \longrightarrow 0, \quad \text { as } n \longrightarrow+\infty,
$$

which is equivalent to

$$
P\left(A_{n}, A_{n+1}\right) \longrightarrow 0, \quad \text { as } n \longrightarrow+\infty \text {. }
$$

Take an arbitrary $\varepsilon>0$. It follows from (2.7) that there exists $k \in \mathbb{N}$ such that

$$
P\left(A_{k}, A_{k+1}\right)<\delta(\varepsilon) .
$$

Without loss of the generality, assume that $\delta(\varepsilon) \leq \varepsilon$ and define the following set:

$$
\Pi:=\left\{A=(x, y, z) \in X^{3}: P\left(\widetilde{F}\left(A_{k}\right), \widetilde{F}(A)\right)<\varepsilon+\delta(\varepsilon), x>x_{k}, y \leq y_{k}, z>z_{k}\right\} .
$$

We claim that

$$
\tilde{F}(A) \in \Pi \quad \forall A \in \Pi \text {. }
$$

Take $A \in \Pi$. Then, by (2.8) and the triangle inequality (which still holds for partial metrics), we have

$$
\begin{aligned}
P\left(A_{k}, \tilde{F}(A)\right)= & \max \left\{p\left(x_{k}, F(x, y, z)\right), p\left(y_{k}, F(y, x, y)\right), p\left(z_{k}, F(z, y, x)\right)\right\} \\
\leq & \max \left\{p\left(x_{k}, x_{k+1}\right)+p\left(x_{k+1}, F(x, y, z)\right), p\left(y_{k}, y_{k+1}\right)\right. \\
& \left.\quad+p\left(y_{k+1}, F(y, x, y)\right), p\left(z_{k}, z_{k+1}\right)+p\left(z_{k+1}, F(z, y, x)\right)\right\} \\
= & \max \left\{p\left(x_{k}, x_{k+1}\right)+p\left(F\left(x_{k}, y_{k}, z_{k}\right), F(x, y, z)\right), p\left(y_{k}, y_{k+1}\right)\right. \\
& \left.\quad+p\left(F\left(y_{k}, x_{k}, y_{k}\right), F(y, x, y)\right), p\left(z_{k}, z_{k+1}\right)+p\left(F\left(z_{k}, y_{k}, x_{k}\right), F(z, y, x)\right)\right\} \\
\leq & P\left(A_{k}, A_{k+1}\right)+P\left(\tilde{F}\left(A_{k}\right), \tilde{F}(A)\right) \\
< & \delta(\varepsilon)+P\left(\widetilde{F}\left(A_{k}\right), \widetilde{F}(A)\right) .
\end{aligned}
$$

We consider the following two cases.

Case $1\left(P\left(A_{k}, A\right) \leq \varepsilon\right)$. By Remark 1.12 and the definition of $\Pi$, the inequality (2.11) turns into

$$
\begin{aligned}
P\left(A_{k}, \tilde{F}(A)\right) & <\delta(\varepsilon)+P\left(\tilde{F}\left(A_{k}\right), \tilde{F}(A)\right) \\
& <\delta(\varepsilon)+P\left(A_{k}, A\right) \\
& <\delta(\varepsilon)+\varepsilon .
\end{aligned}
$$


Case $2\left(\varepsilon<P\left(A_{k}, A\right)<\delta(\varepsilon)+\varepsilon\right)$. That is,

$$
\varepsilon<\max \left\{p\left(x, x_{k}\right), p\left(y, y_{k}\right), p\left(z, z_{k}\right)\right\}<\delta(\varepsilon)+\varepsilon .
$$

Since $x>x_{k}, z>z_{k}, y \leq y_{k}$, then, by (ii), we have

$$
\begin{aligned}
& p\left(F(x, y, z), F\left(x_{k}, y_{k}, z_{k}\right)\right)<\varepsilon \\
& p\left(F(y, x, y), F\left(y_{k}, x_{k}, y_{k}\right)\right)<\varepsilon \\
& p\left(F(z, y, x), F\left(z_{k}, y_{k}, x_{k}\right)\right)<\varepsilon .
\end{aligned}
$$

Hence, combining (2.14) and (2.11), we get

$$
P\left(A_{k}, \tilde{F}(A)\right)<\delta(\varepsilon)+\varepsilon
$$

On the other hand, using (i), one can easily check that

$$
F(x, y, z)>x_{k}, \quad F(y, x, y) \leq y_{k}, \quad F(z, y, x)>z_{k} .
$$

Hence, we conclude that (2.10) holds. By (2.8), we have that $A_{k+1} \in \Pi$, and so, by (2.10) we get

$$
\begin{aligned}
& A_{k+1} \in \Pi \Longrightarrow \tilde{F}\left(A_{k+1}\right)=A_{k+2} \in \Pi \\
& \quad \Longrightarrow \tilde{F}\left(A_{k+2}\right)=A_{k+3} \in \Pi \\
& \quad \ldots \\
& \quad \Longrightarrow A_{n} \in \Pi \quad \forall n>k .
\end{aligned}
$$

Then, for all $n, m>k$, we have

$$
P\left(A_{n}, A_{m}\right) \leq P\left(A_{n}, A_{k}\right)+P\left(A_{k}, A_{m}\right)<2(\varepsilon+\delta(\varepsilon)) \leq 4 \varepsilon .
$$

By definition of $P$, we have

$$
\lim _{n, m \rightarrow \infty} p\left(x_{n}, x_{m}\right)=\lim _{n, m \rightarrow \infty} p\left(y_{n}, y_{m}\right)=\lim _{n, m \rightarrow \infty} p\left(z_{n}, z_{m}\right)=0 .
$$

Consequently, by definition of the metric $d_{p}, d_{p}(x, y) \leq 2 p(x, y)$, so we get

$$
\lim _{n, m \rightarrow \infty} d_{p}\left(x_{n}, x_{m}\right)=\lim _{n, m \rightarrow \infty} d_{p}\left(y_{n}, y_{m}\right)=\lim _{n, m \rightarrow \infty} d_{p}\left(z_{n}, z_{m}\right)=0
$$


Therefore, $\left\{x_{n}\right\},\left\{y_{n}\right\}$, and $\left\{z_{n}\right\}$ are Cauchy sequences in the metric space $\left(X, d_{p}\right)$. Since $(X, p)$ is a complete partial metric space, then, by Lemma $1.2,\left(X, d_{p}\right)$ is also a complete metric space. Hence, there exists a point $(x, y, z) \in X^{3}$ such that

$$
d_{p}\left(x_{n}, x\right), d_{p}\left(y_{n}, y\right), d_{p}\left(z_{n}, z\right) \longrightarrow 0 \quad \text { as } n \longrightarrow+\infty
$$

Again, by Lemma 1.2 and (2.19), we obtain

$$
\begin{gathered}
p(x, x)=\lim _{n \rightarrow \infty} p\left(x_{n}, x\right)=\lim _{n, m \rightarrow \infty} p\left(x_{n}, x_{m}\right)=0, \\
p(y, y)=\lim _{n \rightarrow \infty} p\left(y_{n}, y\right)=\lim _{n, m \rightarrow \infty} p\left(y_{n}, y_{m}\right)=0, \\
p(z, z)=\lim _{n \rightarrow \infty} p\left(z_{n}, z\right)=\lim _{n, m \rightarrow \infty} p\left(z_{n}, z_{m}\right)=0 .
\end{gathered}
$$

We will prove that

$$
F(x, y, z)=x, \quad F(y, x, y)=y, \quad F(z, y, x)=z \text {. }
$$

To this aim, take an arbitrary $\varepsilon>0$. Since

$$
x_{n}=F^{n}\left(x_{0}, y_{0}, z_{0}\right) \longrightarrow x, \quad y_{n}=F^{n}\left(y_{0}, x_{0}, y_{0}\right) \longrightarrow y, \quad z_{n}=F^{n}\left(z_{0}, y_{0}, x_{0}\right) \longrightarrow z
$$

then there exist $n_{1}, n_{2}, n_{3} \in \mathbb{N}$ such that by (2.22)

$$
\begin{gathered}
p\left(x_{l}, x\right)=p\left(F^{l}\left(x_{0}, y_{0}, z_{0}\right), x\right)<p(x, x)+\varepsilon=\varepsilon, \\
p\left(y_{q}, y\right)=p\left(F^{q}\left(y_{0}, x_{0}, y_{0}\right), y\right)<p(y, y)+\varepsilon=\varepsilon, \\
p\left(z_{r}, z\right)=p\left(F^{r}\left(z_{0}, y_{0}, x_{0}\right), z\right)<p(z, z)+\varepsilon=\varepsilon,
\end{gathered}
$$

for all $l \geq n_{1}, q \geq n_{2}, r \geq n_{3}$. Now, taking $n=\max \left\{n_{1}, n_{2}, n_{3}\right\}$ and using Remark 1.12 with the assumption

$$
x_{n}=F^{n}\left(x_{0}, y_{0}, z_{0}\right)<x, \quad y_{n}=F^{n}\left(y_{0}, x_{0}, y_{0}\right)>y, \quad z_{n}=F^{n}\left(z_{0}, y_{0}, x_{0}\right)<z,
$$

by (2.25), we get

$$
\begin{aligned}
p(x, F(x, y, z)) & \leq p\left(x, x_{n+1}\right)+p\left(x_{n+1}, F(x, y, z)\right) \\
& =p\left(x, x_{n+1}\right)+p\left(F^{n+1}\left(x_{0}, y_{0}, z_{0}\right), F(x, y, z)\right) \\
& =p\left(x, x_{n+1}\right)+p\left(F\left(x_{n}, y_{n}, z_{n}\right), F(x, y, z)\right) \\
& <p\left(x, x_{n+1}\right)+\max \left\{p\left(x_{n}, x\right), p\left(y_{n}, y\right), p\left(z_{n}, z\right)\right\} \\
& <2 \varepsilon .
\end{aligned}
$$


Analogously, we get that

$$
p(y, F(y, x, y))<2 \varepsilon, \quad p(z, F(z, y, x))<2 \varepsilon,
$$

which yield that

$$
F(x, y, z)=x, \quad F(y, x, y)=y, \quad F(z, y, x)=z \text {. }
$$

Remark 2.2. Theorem 2.1 remains true if we replace (iv) with one of the following statements.

There exist $x_{0}, y_{0}, z_{0} \in X$ such that

$$
\begin{aligned}
& \text { (1) }\left\{\begin{array}{l}
x_{0} \leq F\left(x_{0}, y_{0}, z_{0}\right), \\
y_{0}>F\left(y_{0}, x_{0}, y_{0}\right), \\
z_{0}<F\left(z_{0}, y_{0}, x_{0}\right),
\end{array}\right. \\
& \text { (2) }\left\{\begin{array}{l}
x_{0} \leq F\left(x_{0}, y_{0}, z_{0}\right), \\
y_{0}>F\left(y_{0}, x_{0}, y_{0}\right), \\
z_{0} \leq F\left(z_{0}, y_{0}, x_{0}\right),
\end{array}\right. \\
& \text { (4) }\left\{\begin{array}{l}
x_{0} \leq F\left(x_{0}, y_{0}, z_{0}\right), \\
y_{0} \geq F\left(y_{0}, x_{0}, y_{0}\right), \\
z_{0}<F\left(z_{0}, y_{0}, x_{0}\right), \\
y_{0} \geq F\left(y_{0}, x_{0}, y_{0}\right), \\
z_{0} \leq F\left(z_{0}, y_{0}, x_{0}\right),
\end{array}\right. \\
& \text { (5) }\left\{\begin{array}{l}
x_{0}<F\left(x_{0}, y_{0}, z_{0}\right), \\
y_{0}>F\left(y_{0}, x_{0}, y_{0}\right), \\
z_{0} \leq F\left(z_{0}, y_{0}, x_{0}\right),
\end{array}\right.
\end{aligned}
$$

\section{Uniqueness of Tripled Fixed Point}

In this section, we will prove the uniqueness of the tripled fixed point.

Theorem 3.1. In addition to hypotheses of Theorem 2.1, assume that, for all $(x, y, z),(u, v, r) \in X^{3}$, there exists $(a, b, c) \in X^{3}$ that is comparable to $(x, y, z)$ and $(u, v, r)$. Then, $F$ has a unique tripled fixed point.

Proof. The set of tripled fixed points of $F$ is not empty due to Theorem 2.1. We suppose that $A=(x, y, z), A^{*}=\left(x^{*}, y^{*}, z^{*}\right) \in X^{3}$ are two tripled fixed points of $F$. We distinguish the following two cases. 
Case 1. $(x, y, z)$ is comparable to $\left(x^{*}, y^{*}, z^{*}\right)$ with respect to the ordering in $X^{3}$, where

$$
\begin{gathered}
\lim _{n \rightarrow+\infty} p\left(F^{n}\left(x_{0}, y_{0}, z_{0}\right), x\right)=p(x, x)=0, \\
\lim _{n \rightarrow+\infty} P\left(F^{n}\left(y_{0}, x_{0}, y_{0}\right), y\right)=p(y, y)=0, \\
\lim _{n \rightarrow+\infty} p\left(F^{n}\left(z_{0}, y_{0}, x_{0}\right), z\right)=p(z, z)=0 .
\end{gathered}
$$

Without loss of the generality, we may assume that

$$
\begin{aligned}
& x=F(x, y, z)<F\left(x^{*}, y^{*}, z^{*}\right)=x^{*}, \\
& y=F(y, x, y) \geq F\left(y^{*}, x^{*}, y^{*}\right)=y^{*}, \\
& z=F(z, y, x)<F\left(z^{*}, y^{*}, x^{*}\right)=z^{*} .
\end{aligned}
$$

By this, definition of $P$, Lemma 1.3, and Remark 1.12, we have

$$
\begin{aligned}
0<P\left(A, A^{*}\right)= & P\left((x, y, z),\left(x^{*}, y^{*}, z^{*}\right)\right) \\
= & \max \left\{p\left(x, x^{*}\right), p\left(y, y^{*}\right), p\left(z, z^{*}\right)\right\} \\
= & \max \left\{p\left(F(x, y, z), F\left(x^{*}, y^{*}, z^{*}\right)\right), p\left(F(y, x, y), F\left(y^{*}, x^{*}, y^{*}\right)\right),\right. \\
& \left.\quad p\left(F(z, y, x), F\left(z^{*}, y^{*}, x^{*}\right)\right)\right\} \\
& <\max \left\{p\left(x, x^{*}\right), p\left(y, y^{*}\right), p\left(z, z^{*}\right)\right\}=P\left(A, A^{*}\right),
\end{aligned}
$$

which is a contradiction and therefore must be $A=A^{*}$.

Case 2. $(x, y, z)$ is not comparable to $\left(x^{*}, y^{*}, z^{*}\right)$. By assumption, there exists $B=(a, b, c) \in X^{3}$ which is comparable to both $A$ and $A^{*}$. Without loss of the generality, we may assume that

$$
\begin{array}{ll}
x=F(x, y, z)<a, & F\left(x^{*}, y^{*}, z^{*}\right)=x^{*}<a, \\
y=F(y, x, y) \geq b, & F\left(y^{*}, x^{*}, y^{*}\right)=y^{*} \geq b, \\
z=F(z, y, x)<c, & F\left(z^{*}, y^{*}, x^{*}\right)=z^{*}<c .
\end{array}
$$

From Proposition 1.14 and (3.4), we have

$$
\begin{aligned}
& \lim _{n \rightarrow+\infty} P\left(\widetilde{F^{n}}(A), \widetilde{F^{n}}(B)\right)=0, \\
& \lim _{n \rightarrow+\infty} P\left(\widetilde{F^{n}}\left(A^{*}\right), \widetilde{F^{n}}(B)\right)=0 .
\end{aligned}
$$


By triangle inequality, we derive

$$
\begin{aligned}
P\left(A, A^{*}\right) & =\lim _{n \rightarrow+\infty} P\left(\widetilde{F^{n}}(A), \widetilde{F^{n}}\left(A^{*}\right)\right) \\
& \leq \lim _{n \rightarrow+\infty} P\left(\widetilde{F^{n}}(A), \widetilde{F^{n}}(B)\right)+\lim _{n \rightarrow+\infty} P\left(\widetilde{F^{n}}(B), \widetilde{F^{n}}\left(A^{*}\right)\right)=0 .
\end{aligned}
$$

By Lemma 1.3, we get $A=A^{*}$.

\section{Results of Integral Type}

Motivated by Suzuki [35] and on the same lines of [31, Theorem 3.1], one can prove the following result.

Theorem 4.1. Let $(X, p, \leq)$ be a partially ordered complete partial metric space, and let $F: X^{3} \rightarrow X$ be a given mapping. Assume that there exists a function $\theta$ from $[0,+\infty)$ into itself satisfying the following:

(I) $\theta(0)=0$ and $\theta(t)>0$ for every $t>0$,

(II) $\theta$ is nondecreasing and right continuous,

(III) for every $\varepsilon>0$, there exists $\delta(\varepsilon)>0$ such that

$$
\varepsilon \leq \theta(\max \{p(x, u), p(y, v), p(z, r)\})<\varepsilon+\delta(\varepsilon) \Longrightarrow \theta(p(F(x, y, z), F(u, v, r)))<\varepsilon,
$$

for all $x \geq u, y \leq v$ and $z \geq r$.

Then, $F$ is a generalized $p$-Meir-Keeler type function.

The following result is an immediate consequence of Theorems 2.1 and 4.1.

Corollary 4.2. Let $(X, p, \leq)$ be a partially ordered complete partial metric space $F: X^{3} \rightarrow X$ be a mapping satisfying the following hypotheses:

(i) F has the mixed strict monotone property,

(ii) for every $\varepsilon>0$, there exists $\delta(\varepsilon)>0$ such that

$$
\varepsilon \leq \int_{0}^{\max \{p(x, u), p(y, v), p(z, r)\}} \phi(t) d t<\varepsilon+\delta(\varepsilon) \Longrightarrow \int_{0}^{p(F(x, y, z), F(u, v, r))} \phi(t) d t<\varepsilon,
$$

for all $x \geq u, y \leq v$ and $z \geq r$, where $\phi:[0,+\infty) \rightarrow[0,+\infty)$ is a locally integrable function satisfying $\int_{0}^{s} \phi(t) d t>0$ for all $s>0$,

(iii) there exist $x_{0}, y_{0}, z_{0} \in X$ such that

$$
x_{0}<F\left(x_{0}, y_{0}, z_{0}\right), \quad y_{0} \geq F\left(y_{0}, x_{0}, y_{0}\right), \quad z_{0}<F\left(z_{0}, y_{0}, x_{0}\right) .
$$

Assume that the hypotheses (a) and (b) given in Theorem 2.1 hold. Then, F has a tripled fixed point. 
To end this paper, we give the following corollary.

Corollary 4.3. Let $(X, d, \leq)$ be a partially ordered complete partial metric space $F: X^{3} \rightarrow X$ be a mapping satisfying the following hypotheses:

(i) F has the mixed strict monotone property,

(ii) for all, $x \geq u, y \leq v$ and $z \geq r$,

$$
\int_{0}^{p(F(x, y, z), F(u, v, r))} \phi(t) d t \leq k \int_{0}^{\max \{p(x, u), p(y, v), p(z, r)\}} \phi(t) d t
$$

where $k \in(0,1)$ and $\phi$ is a locally integrable function from $[0,+\infty)$ into itself satisfying $\int_{0}^{s} \phi(t) d t>0$ for all $s>0$,

(iii) there exist $x_{0}, y_{0}, z_{0} \in X$ such that

$$
x_{0}<F\left(x_{0}, y_{0}, z_{0}\right), \quad y_{0} \geq F\left(y_{0}, x_{0}, y_{0}\right), \quad z_{0}<F\left(z_{0}, y_{0}, x_{0}\right) .
$$

Assume that the hypotheses (a) and (b) of Theorem 2.1 hold. Then, F has a tripled fixed point.

Proof. For all $\varepsilon>0$, we take $\delta(\varepsilon)=(1 / k-1) \varepsilon$ and we apply Corollary 4.2.

Remark 4.4. By taking $\phi(t)=1$, we retrieve the analogous of Theorem 1.8 of Berinde and Borcut on ordered partial metric spaces (with $a=b=c=k / 3$ ). In fact, assume that (1.8) holds for $a=b=c=k / 3$, that is,

$$
p(F(x, y, z), F(u, v, w)) \leq \frac{k}{3}(p(x, u)+p(y, v)+p(z, w))
$$

for all $x \geq u, y \leq v, z \geq w$. From this inequality, we get that

$$
p(F(x, y, z), F(u, v, w)) \leq k \max \{p(x, u), p(y, v), p(z, w)\}
$$

which corresponds to (4.4) with $\phi(t)=1$. Then, we may apply Corollary 4.3.

\section{References}

[1] S. G. Matthews, "Partial metric topology," in Papers on General Topology and Applications (Flushing, NY, 1992), vol. 728, pp. 183-197, Annals of the New York Academy of Sciences, New York, NY, USA, 1994.

[2] R. D. Kopperman, S. G. Matthews, and H. Pajoohesh, "What do partial metrics represent?" in Proceedings of the 19th Summer Conference on Topology and Its Applications, University of CapeTown, 2004.

[3] H. P. A. Künzi, H. Pajoohesh, and M. P. Schellekens, "Partial quasi-metrics," Theoretical Computer Science, vol. 365, no. 3, pp. 237-246, 2006.

[4] T. Abdeljawad, E. Karapnar, and K. Taş, "Existence and uniqueness of a common fixed point on partial metric spaces," Applied Mathematics Letters, vol. 24, no. 11, pp. 1900-1904, 2011.

[5] I. Altun and A. Erduran, "Fixed point theorems for monotone mappings on partial metric spaces," Fixed Point Theory and Applications, vol. 2011, Article ID 508730, 10 pages, 2011. 
[6] I. Altun, F. Sola, and H. Simsek, "Generalized contractions on partial metric spaces," Topology and its Applications, vol. 157, no. 18, pp. 2778-2785, 2010.

[7] H. Aydi, "Some coupled fixed point results on partial metric spaces," International Journal of Mathematics and Mathematical Sciences, Article ID 647091, 11 pages, 2011.

[8] H. Aydi, "Some fixed point results in ordered partial metric spaces," Journal of Nonlinear Science, vol. 4, no. 3, pp. 210-217, 2011.

[9] H. Aydi, "Fixed point results for weakly contractive mappings in ordered partial metric spaces," Journal of Advanced Mathematical Studies, vol. 4, no. 2, pp. 1-12, 2011.

[10] H. Aydi, "Fixed point theorems for generalized weakly contractive condition in ordered partial metric spaces," Journal of Nonlinear Analysis and Optimization, vol. 2, no. 2, pp. 33-48, 2011.

[11] H. Aydi, "Common fixed point results for mappings satisfying $(\psi, \phi)$-weak contractions in ordered partial metric spaces," International Journal of Mathematics and Statistics, vol. 12, no. 2, pp. 53-64, 2012.

[12] H. Aydi, E. Karapınar, and W. Shatanawi, "Coupled fixed point results for $(\psi, \phi)$-weakly contractive condition in ordered partial metric spaces," Computers $\mathcal{E}$ Mathematics with Applications, vol. 62, pp. 4449-4460, 2011.

[13] L. J. Ćirić, B. Samet, H. Aydi, and C. Vetro, "Common fixed points of generalized contractions on partial metric spaces and an application," Applied Mathematics and Computation, vol. 218, no. 6, pp. 2398-2406, 2011.

[14] E. Karapinar, "Weak $\phi$-contraction on partial metric spaces," Journal of Computational Analysis and Applications, vol. 14, no. 2, pp. 206-210, 2012.

[15] E. Karapnar and I. M. Erhan, "Fixed point theorems for operators on partial metric spaces," Applied Mathematics Letters, vol. 24, no. 11, pp. 1894-1899, 2011.

[16] E. Karapinar, "Generalizations of Caristi Kirk's theorem on partial metric spaces," Fixed Point Theory and Applications, vol. 2011, article 4, 2011.

[17] E. Karapınar and U. Yuksel, "Some common fixed point theorems in partial metric spaces," Journal of Applied Mathematics, vol. 2011, Article ID 263621, 17 pages, 2011.

[18] E. Karapinar, "A note on common fixed point theorems in partial metric spaces," Miskolc Mathematical Notes, vol. 12, no. 2, pp. 185-191, 2011.

[19] S. J. O’Neill, “Two topologies are better than one,” Tech. Rep., Univesity of Warwick, 1995.

[20] W. Shatanawi, B. Samet, and M. Abbas, "Coupled fixed point theorems for mixed monotone mappings in ordered partial metric spaces," Mathematical and Computer Modelling, vol. 55, pp. 680687, 2012.

[21] A. C. M. Ran and M. C. B. Reurings, "A fixed point theorem in partially ordered sets and some applications to matrix equations," Proceedings of the American Mathematical Society, vol. 132, no. 5, pp. 1435-1443, 2004.

[22] R. P. Agarwal, M. A. El-Gebeily, and D. O'Regan, "Generalized contractions in partially ordered metric spaces," Applicable Analysis, vol. 87, no. 1, pp. 109-116, 2008.

[23] H. Aydi, "Coincidence and common fixed point results for contraction type maps in partially ordered metric spaces," International Journal of Mathematical Analysis, vol. 5, no. 2, pp. 631-642, 2011.

[24] H. Aydi, M. Postolache, and W. Shatanawi, "Coupled fixed point results for $(\psi, \phi)$-weakly contractive mappings in ordered G-metric spaces," Computers \& Mathematics with Applications, vol. 63, pp. 298309, 2012.

[25] H. Aydi, B. Damjanović, B. Samet, and W. Shatanawi, "Coupled fixed point theorems for nonlinear contractions in partially ordered G-metric spaces," Mathematical and Computer Modelling, vol. 54, pp. 2443-2450, 2011.

[26] T. G. Bhaskar and V. Lakshmikantham, "Fixed point theorems in partially ordered metric spaces and applications," Nonlinear Analysis, vol. 65, no. 7, pp. 1379-1393, 2006.

[27] V. Lakshmikantham and L. Cirić, "Coupled fixed point theorems for nonlinear contractions in partially ordered metric spaces," Nonlinear Analysis, vol. 70, no. 12, pp. 4341-4349, 2009.

[28] N. V. Luong and N. X. Thuan, "Coupled fixed points in partially ordered metric spaces and application," Nonlinear Analysis, vol. 74, no. 3, pp. 983-992, 2011.

[29] H. K. Nashine and B. Samet, "Fixed point results for mappings satisfying $(\psi, \phi)$-weakly contractive condition in partially ordered metric spaces," Nonlinear Analysis, vol. 74, no. 6, pp. 2201-2209, 2011.

[30] J. J. Nieto and R. R. López, "Contractive mapping theorems in partially ordered sets and applications to ordinary differential equations," Order. A Journal on the Theory of Ordered Sets and its Applications, vol. 22, no. 3, pp. 223-239, 2005.

[31] B. Samet, "Coupled fixed point theorems for a generalized Meir-Keeler contraction in partially ordered metric spaces," Nonlinear Analysis, vol. 72, no. 12, pp. 4508-4517, 2010. 
[32] V. Berinde and M. Borcut, "Tripled fixed point theorems for contractive type mappings in partially ordered metric spaces," Nonlinear Analysis, vol. 74, no. 15, pp. 4889-4897, 2011.

[33] K. P. R. Rao and G. N. V. Kishore, "A unique common triple fixed point theorem in partially ordered cone metric spaces," Bulletin of Mathematical Analysis and Applications, vol. 3, no. 4, pp. 213-222, 2011.

[34] H. Aydi, E. Karapinar, and C. Vetro, "Meir-Keeler type contractions for tripled fixed points," preprint.

[35] T. Suzuki, "Meir-Keeler contractions of integral type are still Meir-Keeler contractions," International Journal of Mathematics and Mathematical Sciences, vol. 2007, Article ID 39281, 6 pages, 2007. 


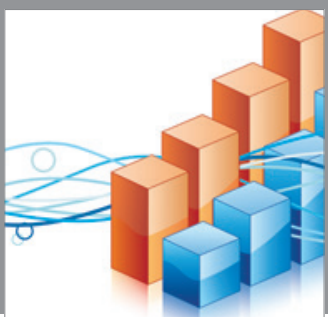

Advances in

Operations Research

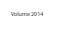

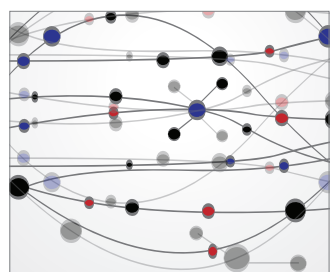

\section{The Scientific} World Journal
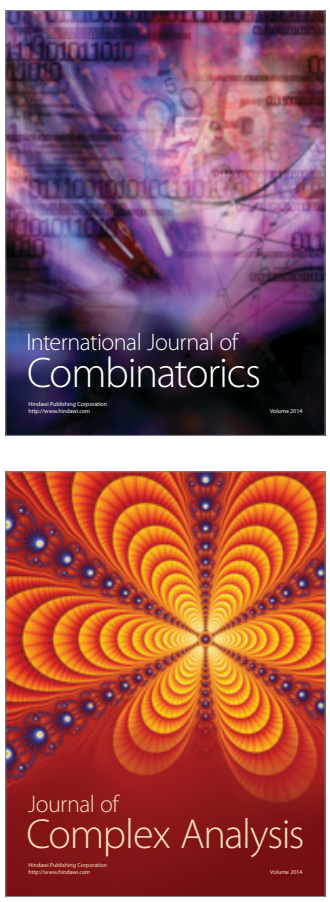

International Journal of

Mathematics and

Mathematical

Sciences
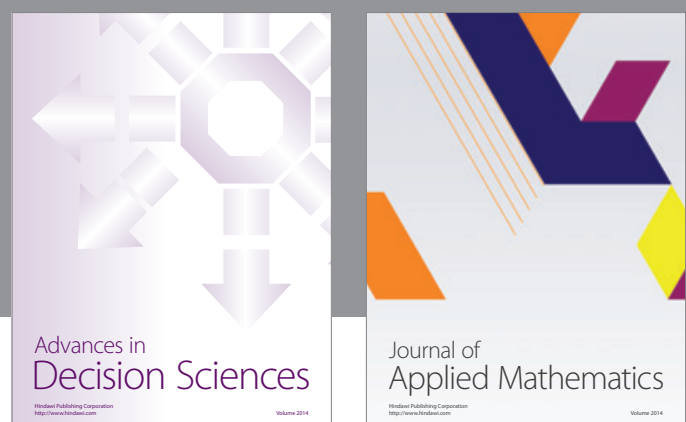

Journal of

Applied Mathematics
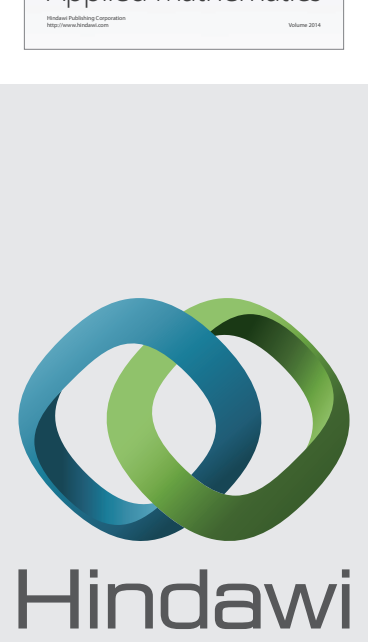

Submit your manuscripts at http://www.hindawi.com
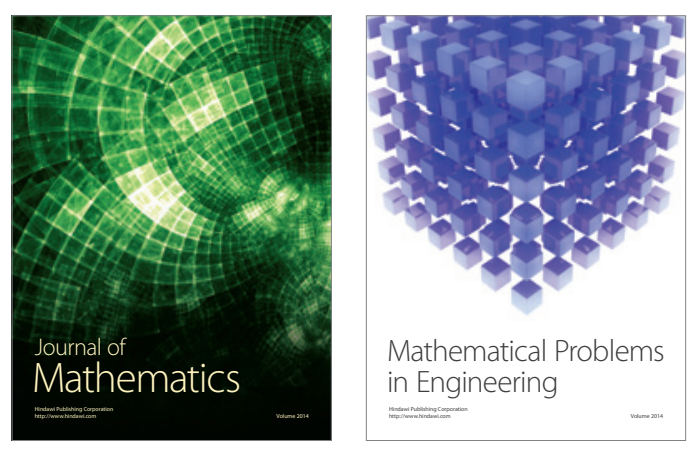

Mathematical Problems in Engineering
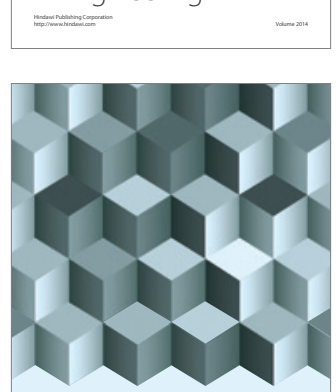

Journal of

Function Spaces
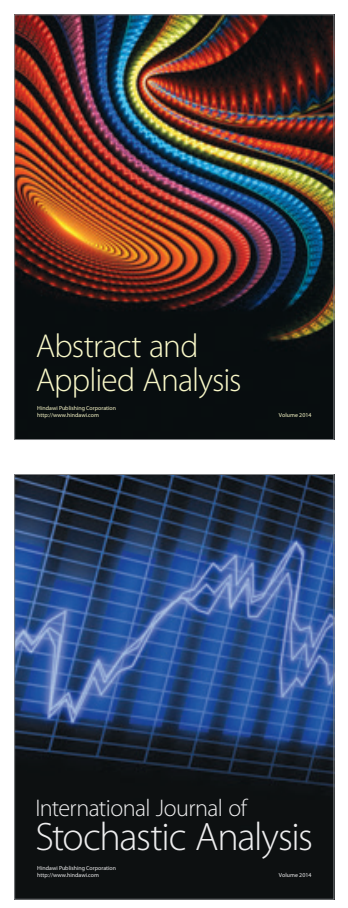

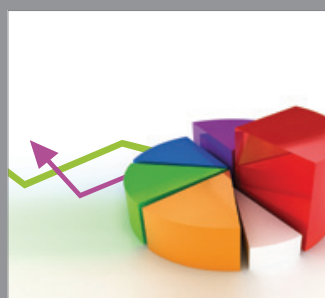

ournal of

Probability and Statistics

Promensencen
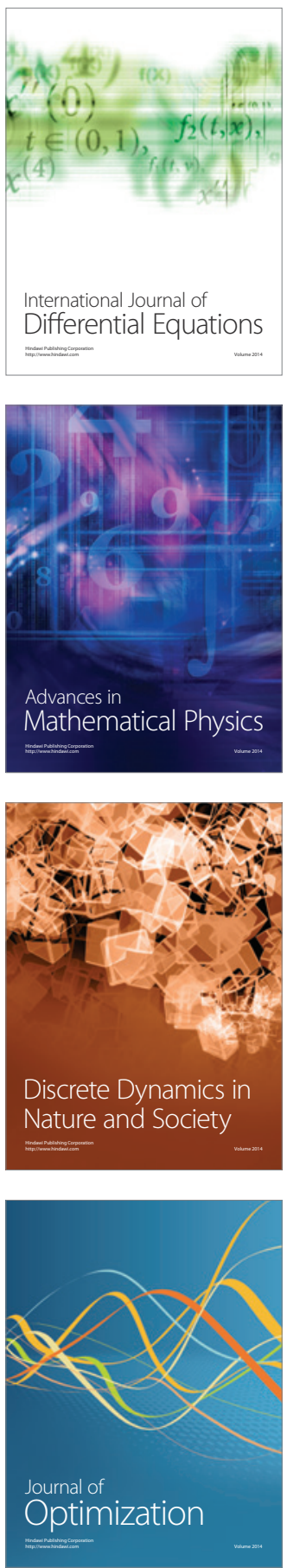